\title{
Photocatalytic Redox Reactions for In-Source Peptide Fragmentation
}

\author{
Liang Qiao, ${ }^{[a]}$ Hongyan Bi, ${ }^{[b]}$ Jean-Marc Busnel, ${ }^{[b]}$ Jerome Waser, ${ }^{[c]}$ Pengyuan Yang, ${ }^{[a]}$ \\ Hubert H. Girault, ${ }^{[b]}$ and Baohong Liu*[a]
}

\begin{abstract}
In-source photocatalytic redox reactions based on a photosensitive target plate have been developed to realize peptide fragmentation during laser desorption ionization. Sample peptides and glucose are simply deposited on a spot of sintered $\mathrm{TiO}_{2}$ nanoparticles. With the irradiation of UV laser on $\mathrm{TiO}_{2}$, electrons are excited from the valence to the conduction band, leaving oxidative holes and reductive electrons to drive various insource redox reactions. Glucose, working here as a hole scavenger and con-
\end{abstract}

ductor, can favor both on-surface reduction and long distance in-plume oxidation, therefore inducing peptide fragmentation. $\mathrm{C}_{\alpha}-\mathrm{C}$ backbone cleavage was observed to generate $a, x$ fragment decay, while the $\mathrm{N}-\mathrm{C}_{\alpha}$ bond cleavage was also sometimes obtained to induce $c, z$ fragmentation, but was rather weaker. The former dissociation is be-

Keywords: mass spectrometry peptides • photocatalysis $\cdot$ redox chemistry $\cdot$ titanium oxide lieved to originate from oxidative routes induced by the valence band holes, based on the oxidation of nitrogen atom at the peptide backbone, including hydrogen-radical abstraction and electron transfer. In contrast, the latter dissociation is supposed to be the result of reductive processes by the conduction band electrons, which are then rather similar to electron capture dissociation in tandem mass spectrometry.

\section{Introduction}

In the past few years, matrix-assisted laser-desorption ionization (MALDI) and related strategies, such as surface-enhanced laser-desorption ionization, have been employed as standard techniques to transfer globally neutral, solid-state samples into gas-phase ions for further analysis by a mass spectrometer. ${ }^{[1-3]}$ Despite of their wide applications in a

[a] L. Qiao, Prof. P. Yang, Prof. B. Liu

Department of Chemistry and Institute of Biomedical Sciences

Fudan University, Shanghai 200433 (China)

Fax: (+86)21-6564-1740

E-mail: bhliu@fudan.edu.cn

[b] H. Bi, Dr. J.-M. Busnel, Prof. H. H. Girault

Laboratoire d'Electrochimie Physique et Analytique

Ecole Polytechnique Fédérale de Lausanne

Station 6, 1015 Lausanne (Switzerland)

Fax: (+41)21-693-3667

E-mail: hubert.girault@epfl.ch

[c] Prof. J. Waser

Laboratoire de Catalyse et de Synthèse Organique

Ecole Polytechnique Fédérale de Lausanne

BCH (Batiment Chimie UNIL), 1015 Lausanne (Switzerland)

Supporting information for this article is available on the WWW under http://dx.doi.org/10.1002/chem.200802229. series of rapid developing areas, such as proteomics, the use of these ionization methods has progressed mainly in an empirical manner ${ }^{[4,5]}$ Even if the ablation process is well understood, the different charge-transfer reactions that take place are still a matter of discussion. ${ }^{[5]}$ The major problem is that MALDI is a very complex photoelectrochemical event happening within a few nanoseconds. Under the strong illumination of UV laser, a dense plume containing matrix and sample neutrals as well as reactive species, such as radicals, electrons, and hydrogen atoms is formed and expanded into the vacuum of the mass spectrometer ion source, ${ }^{[4,6]}$ in which in-source reactions can happen.

Despite being used as a non-destructive ionization technique, the investigation of in-source reactions in MALDI can also be very interesting and further open the way to some new applications. For example, recently we have shown that $\mathrm{TiO}_{2}$ nanoparticles can be used to modify target plates to carry out efficient in-source photocatalytic reactions during the MALDI procedure. The employed semiconductor photochemistry principle has already been applied to many fields. ${ }^{[7-10]}$ Specifically, under the UV-laser irradiation, electrons are excited from the valence band to the conduction band of $\mathrm{TiO}_{2}$ nanoparticles, ${ }^{[1-13]}$ leaving oxidative holes to drive in-source oxidation reactions and reductive electrons to induce in-source reduction reactions. As an 
application of the oxidative principle, on-line peptide tagging has been realized by adding hydroquinone, which is first oxidized to benzoquinone by the photogenerated valence band holes and then reacts with the cysteinyl peptide to form a complex through Michael addition. ${ }^{[14]}$ As an application of the reductive principle, an in-source cleavage of disulfide bond was achieved with the assistance of electrondonor reagents, in which the disulfide bonds are directly reduced by the photogenerated conduction band electrons. ${ }^{[15]}$

In this paper, we demonstrate that the concept of $\mathrm{TiO}_{2}$-induced in-source photocatalytic redox reactions can be further applied to realize sample dissociation along the peptide backbone, either directly or with the participation of intermediate molecules. By depositing peptides and glucose on the mesoporous $\mathrm{TiO}_{2}$-modified photosensitive target plate, fragmentation was easily observed during laser desorption ionization (LDI) without the assistance of any conventional organic matrix. Here, glucose can be considered as a very efficient electron donor, ${ }^{[16]}$ working as a hole scavenger or conductor to help either reduction on the surface of the $\mathrm{TiO}_{2}$ layer or long-distance oxidation in the plume. After scavenging holes, the electron quenching from on-surface or in-volume recombination can be greatly prevented, ${ }^{[13]}$ therefore releasing more free electrons for in-source reduction reactions, while the generated oxidized glucose can further oxidize other present species, thus realizing long-distance inplume oxidation reactions.

Intense $\mathrm{C}_{\alpha}-\mathrm{C}$ backbone cleavage is observed here by using this proposed in-source photocatalytic peptide fragmentation, which is otherwise rarely obtained: the novel $\mathrm{C}_{\alpha}-\mathrm{C}$ backbone cleavages have only been reported as being dominant upon photodissociation of singly charged cations with UV light at $157 \mathrm{~nm}^{[17,18]}$ or from the electron-detachment dissociation (EDD) of polypeptide polyanions. ${ }^{[19-21]}$ Indeed, the most widely employed peptide dissociation strategies mainly generate $b, y$ or $c, z$ dissociation. ${ }^{[22-25]}$ Here, novel oxidative routes were supposed to explain the unusual fragmentation pattern. Meanwhile, $\mathrm{N}-\mathrm{C}_{\alpha}$ backbone cleavage is also observed sometimes though much weaker, which can be from a reductive process induced by the conduction band electrons, rather similar to the electron capture dissociation $(E C D)^{[22]}$ in tandem mass spectrometry. The presented insource peptide fragmentation can be of interest for studying photoelectrochemical-controlled reactions during laser desorption ionization.

\section{Results and Discussion}

Photocatalytic peptide fragmentation on a $\mathrm{TiO}_{2}$ photoelelctrode plate: The photosensitive target plate was fabricated by using $\mathrm{P} 25 \mathrm{TiO}_{2}$ nanoparticles. ${ }^{[14]}$ Scheme 1 schematically illustrates the structure of this functional plate, including a polished steel plate covered by an array of sintered $\mathrm{TiO}_{2}$ nanoparticle spots. Peptides and the electron donor/hole conductor molecules are deposited on the mesoporous spot. Under the irradiation of a UV laser, each spot can behave

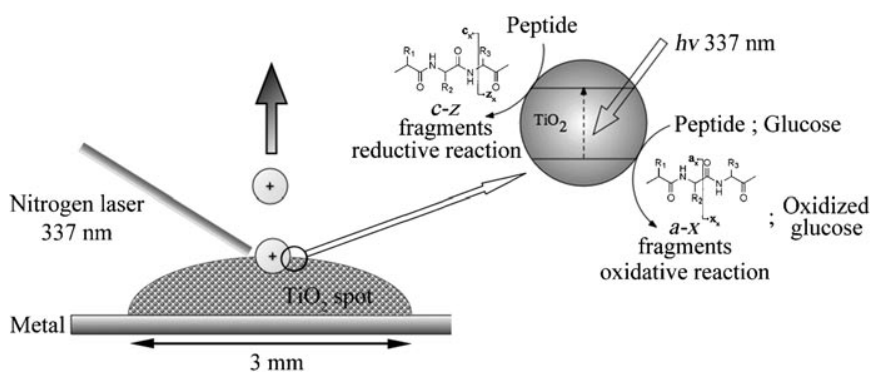

Scheme 1. Schematic representation of the $\mathrm{TiO}_{2}$-induced in-source redox reactions for peptide fragmentation.

as a photoelectrode as in a dye-sensitized solar cell $;^{[10]}$ and a series of photocatalytic reactions are then induced by the photosensitized $\mathrm{TiO}_{2}$ nanoparticles, which can drive peptide in-source dissociation. The resulting ionized fragments can then be accelerated by the electric field after a short delay period and separated in the TOF analyzer. It should be stressed that, as in a dye-sensitized solar cell, the nanoporous $\mathrm{TiO}_{2}$ offers an excellent photon-capture cross section, and a very large surface/volume ratio such that most of the molecules present on and in the spot can undergo photoelectrochemical reactions very efficiently.

Figure $1 \mathrm{a}$ shows the fragmentation result of angiotensin I obtained on the $\mathrm{TiO}_{2}$ photoelectrode plate by using L-glucose as an electron donor/hole conductor in positive-ion
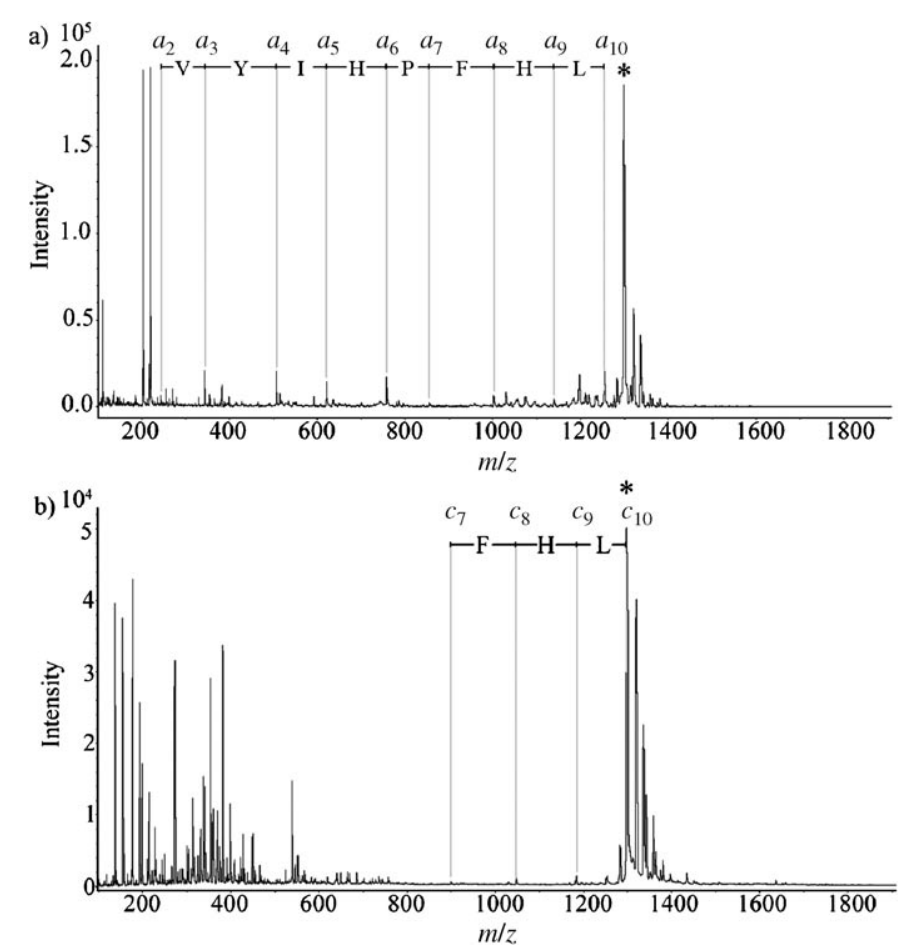

Figure 1. Fragmentation result of angiotensin $\mathrm{I}(\approx 70 \mathrm{pmol})$ obtained a) on a $\mathrm{TiO}_{2}$ photoelectrode plate in the presence of L-glucose, and b) on a normal ground-steel plate using a DHB matrix in the positive-ion mode with ion extraction delay $(600 \mathrm{~ns}) . *$-tagged peaks correspond to parent ions and $a_{x}$ - or $c_{x}$-tagged peaks correspond to $a_{x}$ or $c_{x}$ fragments, respectively. 
mode with an ion extraction delay $(600 \mathrm{~ns})$. A complete series of $a_{n}$ fragments starting from $a_{2}$ were detected with rather clean background in low mass range, while almost no $c_{n}$ fragments were observed. To illustrate the unique characteristic of the dissociation pattern, in-source fragmentation of angiotensin I (DRVYIHPFHL) was performed on a normal ground-steel target plate by using a 2,5-dihydroxybenzoic acid (DHB) matrix with ion extraction delay (600 ns) in the positive-ion mode. Unlike the $\mathrm{TiO}_{2}$-induced peptide fragmentation, only several weak $c_{n}$ fragments were obtained, and due to the presence of organic matrix, it is nearly impossible to read out fragments with $\mathrm{m} / \mathrm{z}$ less than 700 , as shown in Figure $1 \mathrm{~b}$. To further validate the unusual decay pattern, a longer peptide, oxidized $\beta$-insulin $(7 \mathrm{pmol}$, FVNQHLCoxGSHLVEALYLVCoxGERGFFYTPKA), ${ }^{[26]}$ was used, and the $\mathrm{TiO}_{2}$-induced photocatalytic peptide fragmentation was performed in the negative mode. With L-glucose as the electron donor/hole conductor and an ion extraction delay ( $600 \mathrm{~ns})$, strong $a, x$ decay was observed and a complete $a_{n}$ fragments list from $a_{7}$ to $a_{18}$ was obtained, as shown in Figure $2 \mathrm{a}$. The $c_{n}$ fragment series was also detected, but with weaker signal intensities. The control experiment was performed on a normal ground-steel plate with a DHB matrix in the negative-ion mode with an ion extraction delay $(600 \mathrm{~ns})$. Similar to the previously reported results, ${ }^{[26]}$ only $c_{n}$ fragments were effectively obtained, as shown in Figure $2 \mathrm{~b}$. It should also be mentioned that the fragment-to-parent-peak area ratio seen in Figure $2 \mathrm{a}$ is
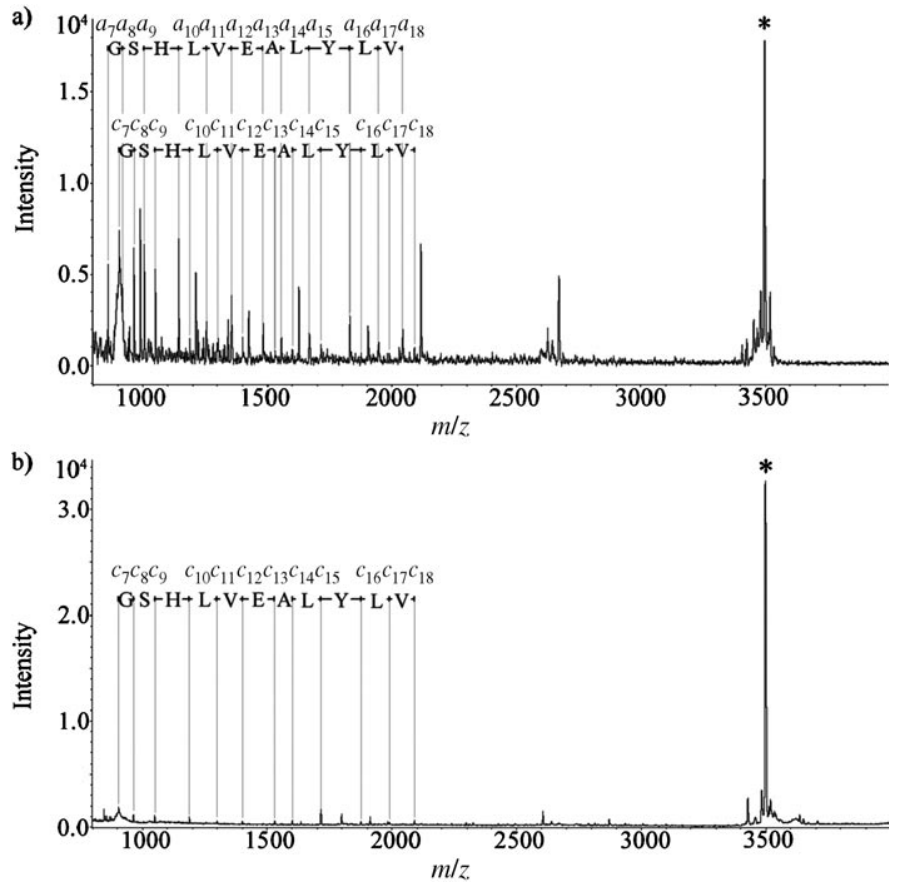

Figure 2. Fragmentation result of oxidized $\beta$-insulin $(\approx 7 \mathrm{pmol})$ obtained a) on a $\mathrm{TiO}_{2}$ photoelectrode plate in the presence of L-glucose, and b) on a normal ground-steel plate using a DHB matrix in the negative-ion mode with ion extraction delay $(600 \mathrm{~ns})$. *-tagged peaks correspond to parent ions and $a_{x}$ - or $c_{x}$-tagged peaks correspond to $a_{x}$ or $c_{x}$ fragments, respectively. much bigger than that observed in Figure $2 b$, indicating a more intense dissociation. From an electrochemical viewpoint, it is interesting to consider what happens during the application of the electric field. Indeed, in the positive mode the $\mathrm{TiO}_{2}$-modified plate acts as an anode, and the electrons stored in the nanoparticles diffuse to the steel plate, thereby resetting the mesoporous structure for the subsequent laser shot. Alternatively, in the negative mode, the plate acts as a cathode.

Electron donors: Glucose plays an important role in the $\mathrm{TiO}_{2}$-induced photocatalytic peptide fragmentation. Except some basic functions, such as separating sample molecules, transporting energy and assisting protonation, it mainly works here as a hole scavenger or conductor for either freeing more electrons to promote reduction reactions on the surface of the $\mathrm{TiO}_{2}$ layer or working as an intermediate reagent to realize long-distance in-plume oxidation reactions. Indeed, alcohol, glucose, and other sugars are commonly used to supply electrons or capture holes on $\mathrm{TiO}_{2}$ in photoelectrochemistry. ${ }^{[16]}$ Under the UV radiation, the glucose can inject electrons to the valence band of the $\mathrm{TiO}_{2}$ and many oxidative radicals, for example, $\left[\mathrm{RCH}_{2} \mathrm{O}^{*}\right]$, [RCOO$]$ and [ $\left.\mathrm{RCO}^{*}\right]$ can be generated on the surface of the mesoporous structure, ${ }^{[27]}$ which can either undergo further in-source reactions with each other to form complexes or work as initial reactive species to oxidize other samples, for example peptides in this work.

Figure 3 a shows the mass spectrum of L-Glucose obtained on the $\mathrm{TiO}_{2}$ photoelectrode plate in positive-ion mode with
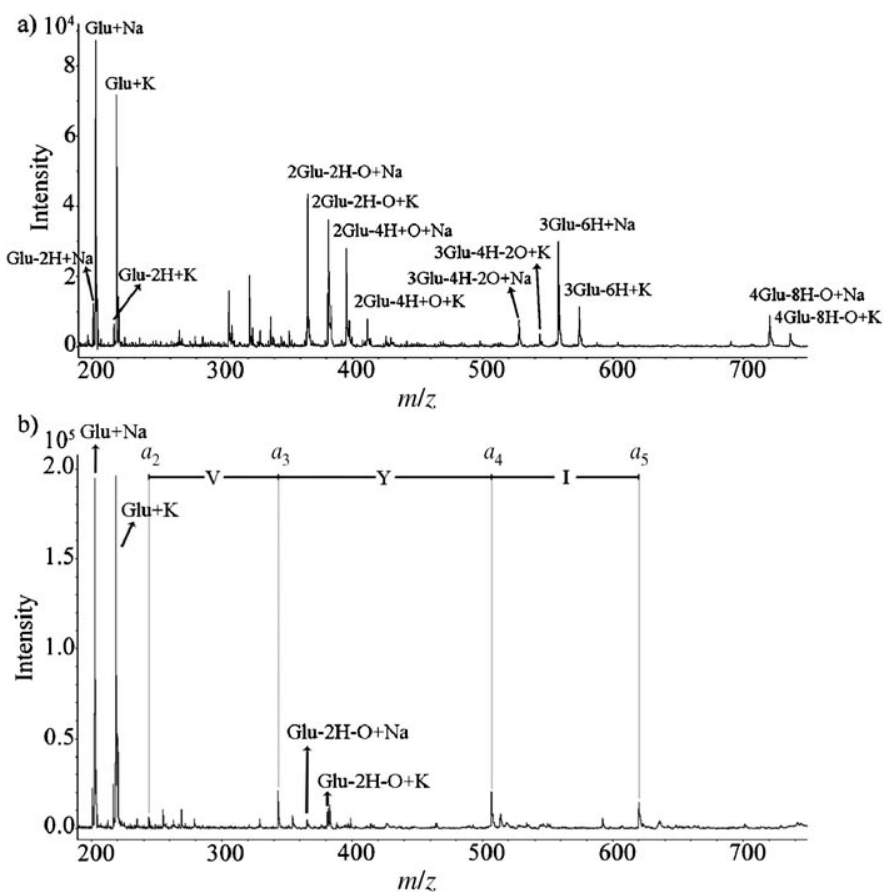

Figure 3. a) Mass spectrum of L-glucose $(56 \mathrm{nmol})$ and b) partial mass spectrum of L-glucose $(56 \mathrm{nmol})$ in the presence of angiotensin I $(\approx 70 \mathrm{pmol})$ obtained by using the $\mathrm{TiO}_{2}$ plate in the positive-ion mode with the ion extraction delay (600 ns). 
an ion extraction delay (600 ns). The amount of glucose used was equal to that employed in the peptide fragmentation experiments, which was determined after a brief optimization. Though it is supposed that neutral oxidative glucose radicals $\left[\mathrm{RCH}_{2} \mathrm{O}^{\circ}\right],\left[\mathrm{RCOO}^{\circ}\right]$, and [ $\left.\mathrm{RCO}^{\circ}\right]$ are generated on the $\mathrm{TiO}_{2}$ layer, the high reactivity makes them rather unstable, and thus can not be detected directly. On the mass spectrum, many peaks for the products that may be from the secondary self-reaction of these oxidative radicals are observed, including oxidized glucose of [glucose-2H], which is obtained from $\left[\mathrm{RCH}_{2} \mathrm{O}^{\circ}\right]$ by losing $\left[\mathrm{H}^{*}\right]$, and complexes of [2 glucose $-2 \mathrm{H}-\mathrm{O}], \quad[2$ glucose $-4 \mathrm{H}+\mathrm{O}], \quad$ [3 glucose $-4 \mathrm{H}-$ $2 \mathrm{O}],[3$ glucose $-2 \mathrm{H}]$ and $[4$ glucose $-8 \mathrm{H}-\mathrm{O}]$, which are from the combination of two or more oxidative radicals. However, with the addition of peptides, most of these signals are absent when $a$ fragments from the peptide appear on the mass spectrum (Figure $3 \mathrm{~b}$ ), indicating that the photogenerated oxidative radicals are playing a role in the $\mathrm{C}_{\alpha}-\mathrm{C}$ backbone cleavage process. It can be proposed that the generation of glucose radical is initiated by the photogenerated holes either on the surface or in the nanopores of the $\mathrm{TiO}_{2}$ layer, while the secondary reaction between oxidative glucose radicals and peptides would happen in-plume, therefore restricting self-reactions of the radicals. Otherwise, the peptide fragmentation may also directly be induced by the photogenerated holes, thereby preventing the oxidation of glucose as a competitor. To further illustrate this principle, several other hole scavengers, for example, $\alpha$-D-glucose, $\alpha$-lactose, D-sucrose, D-galactal, D-glucal and citric acid, were also used as intermediate molecules in the $\mathrm{TiO}_{2}$-induced photocatalytic peptide fragmentation strategy. All these compounds were found to have the ability to work as hole scavengers/conductors. However, although all of the tested compounds allowed the observation of peptide fragmentations, the required amounts of peptide varied from one compound to the other. It is supposed to be a consequence of the different electron-donating abilities of these reagents (see Supporting Information for further details). ${ }^{[16]}$

In-source photooxidation-induced dissociation: To reach a stable state, the oxidative glucose radicals, $\left[\mathrm{RCH}_{2} \mathrm{O}^{\circ}\right]$, [RCOO$\left.{ }^{\circ}\right]$ and [RCO'], generated on the $\mathrm{TiO}_{2}$ mesoporous structure would either capture a hydrogen radical or an electron from the peptide, which would therefore be oxidized. As the fragmentation happens randomly between every two amino acids, the oxidation reaction probably takes place at the backbone of the peptides rather than at special side chains. Considering the structure of the peptides backbone, which is made up of amide groups and saturated carbon atoms, the amide nitrogen is most likely to be oxidized compared with the carbonyl oxygen and backbone carbon atoms. Here, we consider two oxidation mechanisms to interpret the peptide $a, x$ cleavage, a hydrogen-radical abstraction and an electron transfer, as illustrated in Scheme $2 \mathrm{a}$ and $\mathrm{b}$. In the first scenario, a hydrogen atom is captured by the glucose radical from the amide, leaving a reactive radical on the nitrogen atom. Subsequent radical-initi-

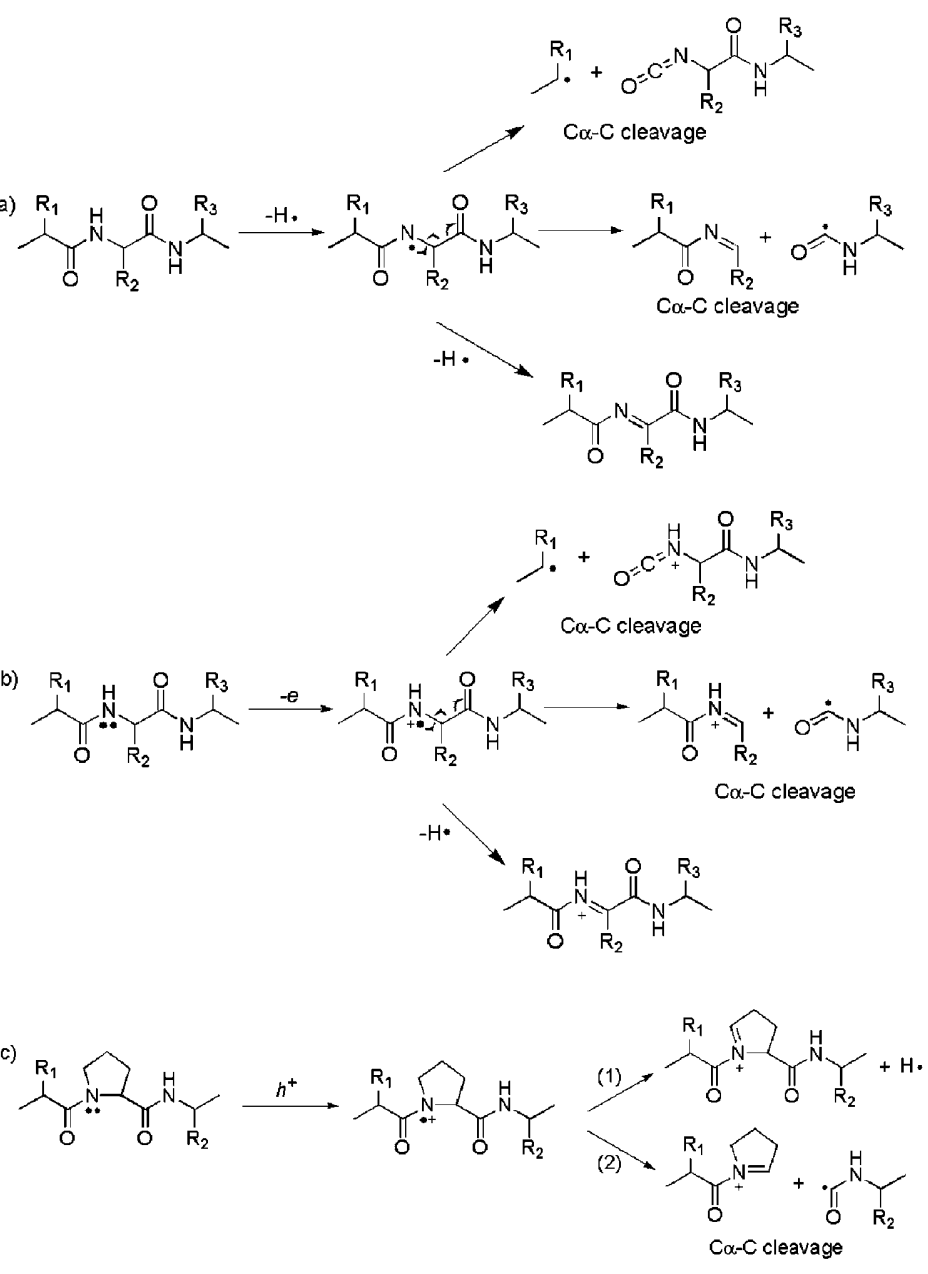

Scheme 2. Supposed mechanisms for the $\mathrm{C}_{\alpha}-\mathrm{C}$ bond cleavage during $\mathrm{TiO}_{2}$-induced photocatalytic peptide fragmentation. a) Hydrogen-radicalabstraction mechanism and b) electron-transfer mechanism. c) Supposed mechanism for the backbone cleavage of $\mathrm{C}_{\alpha}-\mathrm{C}$ bond N-terminal to proline residue during $\mathrm{TiO}_{2}$-induced photocatalytic peptide fragmentation.

ated fragmentation can proceed through hydrogen atom elimination leading to the oxidation to the imine derivative. Alternatively, $\mathrm{C}_{\alpha}-\mathrm{C}$ bond cleavage happens, which corresponds to an $a, x$ decay. The ionization of the generated $a$ and $x$ fragments may happen subsequently or the peptides may be already ionized before the fragmentation. In the second scenario, the only difference is that electrons are captured by the oxidizing glucose radicals from the amide nitrogen atom instead of $\mathrm{H}$ atoms, thus generating positively charged nitrogen radicals at the peptide backbone, leading to the $\mathrm{C}_{\alpha}-\mathrm{C}$ backbone cleavage.

Both the H-radical-abstraction and electron-transfer mechanisms can justify the results obtained. Indeed, the $\mathrm{H}$ radical-abstraction path is quite similar to the route reported in the EDD of polypeptide polyanions, which is also an oxidative process by losing electrons. ${ }^{[19-21]}$ However, the electron-transfer one can be reasonable as complementary route and more or less plausible. Based on the literature, the hydrogen atom at the $\alpha$-carbon of the peptide backbone is more labile as compared to that of the amide. ${ }^{[28,29]}$ More- 
over, it has been reported in organic electrochemistry that the mechanism for the anodic $\alpha$-oxidation of amides starts with a one-electron oxidation to form the radical cation at the nitrogen atom. ${ }^{[30-33]} \mathrm{A}$ careful analysis of our own results suggests that the electron-transfer mechanism is at least one of the routes that exist in the $\mathrm{TiO}_{2}$-induced photocatalytic peptide $\mathrm{C}_{\alpha}-\mathrm{C}$ bond cleavage. Indeed, as reported in the case of $\mathrm{EDD},{ }^{[19]}$ taking into account the hydrogen-radical-abstraction mechanism, it would not be possible to obtain fragmentation for amino acid residues that do not bear hydrogen atom on the amino group, such as proline. Thus, the $a_{7}$ fragment originating from the cleavage between proline and phenylalanine in angiotensin I (DRVYIHPFHL) should be absent. However, the $a_{7}$ ion was indeed observed though much weaker than others (Figure 4), which demonstrates

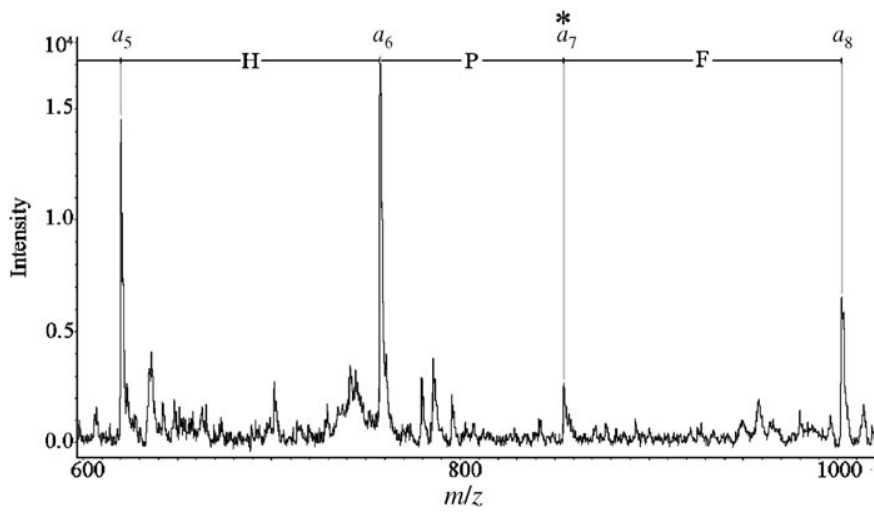

Figure 4. Partial mass spectrum of angiotensin $\mathrm{I}(\approx 70 \mathrm{pmol})$ in the presence of L-glucose $(56 \mathrm{nmol})$ obtained by using the $\mathrm{TiO}_{2}$ plate in the positive-ion mode with the ion extraction delay $(600 \mathrm{~ns})$.

that the hydrogen-abstraction mechanism cannot be the only approach existing during the oxidative $a, x$ fragmentation. In contrast, the electron-transfer mechanism can interpret this phenomenon quite well, as shown in Scheme $2 \mathrm{c}$. After losing an electron, the nitrogen radical cation is generated at the peptide backbone, and may further lead to the radical fragmentation reaction to generate $\mathrm{C}_{\alpha}-\mathrm{C}$ bond cleavage. A second $\alpha$-hydrogen elimination pathway is also possible, which could explain the lower intensities observed.

Generally, the two possible mechanisms can be considered to explain the oxidative $\mathrm{C}_{\alpha}-\mathrm{C}$ backbone cleavage. The details of these in-source reactions are likely to be very complex, but the oxidation mechanism proposed above can explain the observed phenomena reasonably well. One may argue that the $a$ ions can also originate from a secondary dissociation of $c$ ions or energetic activation. In these cases, stronger $c$ or $b$ ions should be undoubtedly observed, but are found to be absent in these experimental conditions. To further demonstrate that the oxidative radicals generated from the photoelectrochemical processes can really drive $\mathrm{C}_{\alpha}-\mathrm{C}$ backbone cleavages, an oxidative radical initiator, $\mathrm{N}$ hydroxyphthalimide ${ }^{[34,35]}$ was employed as an additive to the DHB matrix, while other experimental conditions remained as for the experiment shown in Figure $2 b$ to perform in- source fragmentation of oxidized $\beta$-insulin. As a consequence, a new series of $a$ fragments can be observed with the presence of the oxidative radical initiator, Figure 5.
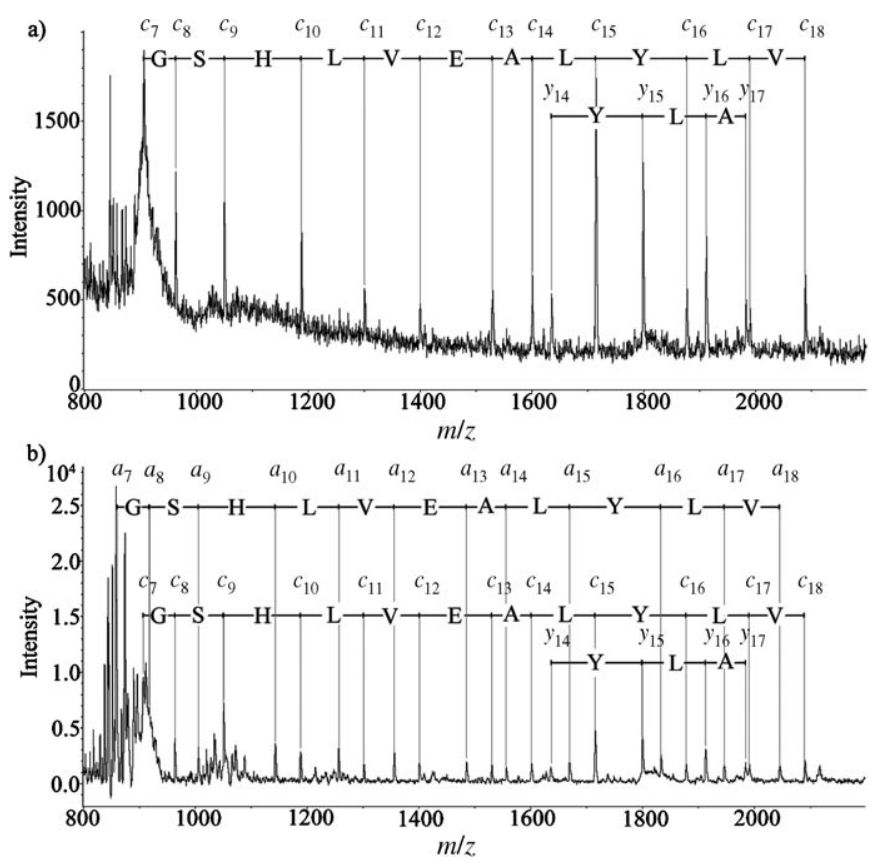

Figure 5. Zoomed fragmentation pattern of oxidized $\beta$-insulin $(\approx 7 \mathrm{pmol})$ obtained on a normal ground-steel plate with a) a DHB matrix and b) a $N$-hydroxyphthalimide/DHB matrix in the positive-ion mode with ion extraction delay $(600 \mathrm{~ns}) .{ }^{*}$-tagged peaks correspond to parent ions and $a_{x^{-}}$ or $c_{x}$-tagged peaks correspond to $a_{x}$ or $c_{x}$ fragments, respectively.

Under the UV-laser energy, $N$-hydroxyphthalimide can very easily donate a hydrogen atom and therefore forms a [RO'] radical, which is indeed rather similar to that generated from glucose on the $\mathrm{TiO}_{2}$ semiconductor under laser radiation. Thus, it appears credible to propose that the oxidative [RO'] radicals have the ability to induce $a, x$ fragmentation through an oxidative route.

In-source photoreduction-induced dissociation: Besides the unusual $a_{n}$ fragments, a series of $c_{n}$ fragments were also observed, though with much weaker intensities in the case of the $\mathrm{TiO}_{2}$-induced photocatalytic peptide fragmentation of oxidized $\beta$-insulin, Figure $2 \mathrm{a}$. Under the UV-laser irradiation, both oxidative holes and reductive electrons are generated from the semiconductor $\mathrm{TiO}_{2}$ nanoparticles. As illustrated in Scheme 1, the holes can be employed to perform peptide $a, x$ cleavage by oxidizing glucose, whilst the electrons would induce $c, z$ fragmentation through an approach that might be similar to the ECD procedure, depicted on Scheme 3. However, it has been reported that the trapping of conduction band electrons on the nanoparticles is much faster than that of the valence band holes, ${ }^{[36]}$ and as a result, few electrons are available for reducing reactions. Besides, there is also a lack of an electron conductor to help long-distance reduction. Thus, the intensities of the $c_{n}$ ions signals 


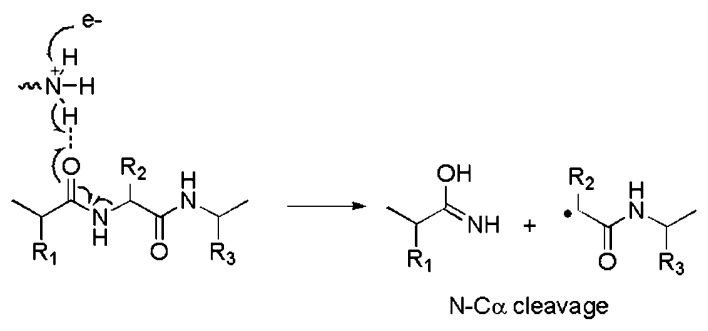

Scheme 3. Supposed mechanism for the $\mathrm{N}-\mathrm{C}_{\alpha}$ bond cleavage during $\mathrm{TiO}_{2}$-induced photocatalytic peptide fragmentation.

are much weaker, and for the relatively less efficient photocatalytic fragmentation of angiotensin I, the phenomenon ultimately disappeared, therefore leaving an unusual peptide decay pattern of $a, x$ dissociation. This condition may be changed by for example adding molecules favoring electron hopping to realize long-distance reduction dissociation.

\section{Conclusions}

In summary, we have developed in-source photocatalytic redox reactions to realize peptide fragmentation by performing $\mathrm{LDI}$ on a $\mathrm{TiO}_{2}$-derived plate with the assistance of glucose, in which both oxidative $\mathrm{C}_{\alpha}-\mathrm{C}$ backbone cleavage and reductive $\mathrm{N}-\mathrm{C}_{\alpha}$ bond cleavage were observed. Although the sensitivity is still not enough to have practical applications in proteomics, we have demonstrated that photocatalytic reactions offer new patterns of peptide sequencing. With the unusual in-source fragmentation pattern of $a, x$ dissociation, the on-line photocatalytic peptide dissociation can be interesting for mechanistic studies of photoelectrochemical ionization occurring on $\mathrm{TiO}_{2}$ nanoparticles. Moreover, with the in situ oxidation and reduction properties, the photosensitive plate may be employed for the measurements of protein oxidation and antioxidant capabilities.

\section{Experimental Section}

Materials: Titanium dioxide nanoparticles were obtained from Degussa (P25, Germany). Ethanol (99.8\%), acetic acid (99.5\%), and acetonitrile $(99.5 \%)$ were obtained from Fluka (Germany). Trifluoroacetic acid (TFA, 99\%) and D-galactal (95\%) were purchased from Acros organics (Belgium). 2,5-Dihydroxybenzoic acid (DHB, $98 \%$ ), L-glucose (98\%), $\alpha$ D-glucose $(98 \%), \alpha$-lactose (reagent grade), D-sucrose (99\%), D-glucal $(96 \%)$, citric acid $(99 \%), N$-hydroxyphthalimide (97\%), and $\beta$-insulin, oxidized from bovine insulin $(90 \%)$, were obtained from Sigma-Aldrich (Germany). Angiotensin I (98\%) was purchased from Bachem (Switzerland). All these reagents were used as received without further purification. Deionized water $(18.2 \mathrm{M} \Omega \mathrm{cm})$ used for all experiments was obtained from a Milli-Q system (Millipore, Bedford, MA, USA).

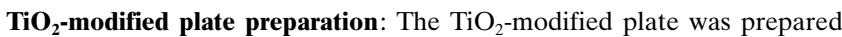
according to a previous report. ${ }^{[37]}$ To remove impurity and decrease aggregation, the commercial $\mathrm{P} 25 \mathrm{TiO}_{2}$ nanoparticles were heated (at $300^{\circ} \mathrm{C}$ for $2 \mathrm{~h}$ ) and then ground in a mortar (for $2 \mathrm{~h}$ ). A stable suspension $\left(100 \mathrm{mgml}^{-1}\right)$ was obtained by separating the nanoparticles in ethanol $(89 \%)$ and kept as the stock solution. For the fabrication of $\mathrm{TiO}_{2}$-modified plates, the solution was diluted in deionized water to a $\mathrm{TiO}_{2}$ concen- tration of $4 \mathrm{mg} \mathrm{mL}^{-1}$, dropped on a commercial polished steel plate for a Bruker Microflex mass spectrometer as an array of spots $(\approx 2 \mu \mathrm{L})$ and dried in ambient conditions. The resulting modified plate was subsequently heated in an oven. The oven temperature rose from room temperature to $400^{\circ} \mathrm{C}$ in $2 \mathrm{~h}$, stayed at $400^{\circ} \mathrm{C}$ for one hour, cooled down to $60^{\circ} \mathrm{C}$ in $9 \mathrm{~h}$ and then kept at this temperature until use.

MS method and data analysis: An aqueous solution of the peptide $(1 \mu \mathrm{L})$ was deposited on the prepared $\mathrm{TiO}_{2}$-modified plate and dried at room temperature and atmosphere (for $\approx 10 \mathrm{~min}$ ). After drying, an aqueous solution of L-glucose $\left(1 \mu \mathrm{L}, 10 \mathrm{mgml}^{-1}\right)$ or a solution of other sugars $(1 \mu \mathrm{L}$, $\left.10 \mathrm{mgml}^{-1}\right)$ or citric acid $\left(1 \mu \mathrm{L}, 10 \mathrm{mgml}^{-1}\right)$ was dropped and then dried under the same conditions before being subjected to LDI-TOF-MS. For the control experiments, an aqueous solution of peptide $(1 \mu \mathrm{L})$ was deposited on a normal ground-steel plate and dried in ambient conditions for $\approx 10 \mathrm{~min}$ followed by the addition of a DHB matrix $[1 \mu \mathrm{L}, \mathrm{DHB}$ $\left(10 \mathrm{mgml}^{-1}\right)$ in acetonitrile $(50 \%)$, TFA $(0.1 \%)$ and deionized water (49.9\%)] or a $N$-hydroxyphthalimide/DHB matrix $[1 \mu \mathrm{L}, N$-hydroxyphthalimide $\left(5 \mathrm{mgml}^{-1}\right)$ and DHB $\left(10 \mathrm{mgml}^{-1}\right)$ in acetonitrile $(50 \%)$, TFA $(0.1 \%)$ and deionized water $(49.9 \%)]$. All the LDI-TOF or MALDITOF mass spectrometry experiments were performed on a Bruker Microflex equipped with a nitrogen laser operated at $337 \mathrm{~nm}$. For investigating in-source reactions, an optimized ion extraction delay of $600 \mathrm{~ns}$ was employed to favor the secondary in-plume reactions. The laser intensity was adjusted to $10 \%$ above threshold to supply enough energy for inducing the reactions. The data analysis and peptide sequence were performed using the flexAnalysis software from Bruker.

\section{Acknowledgements}

This work is supported by NSFC (20775016, 20735005), Swiss NSF grant "Development for new analytical tools for proteomics" (200020-105489), 973 Program (2007CB714506), Shuguang 06SG02 and the Swiss-China research program. B.H.L. is grateful to EPFL for a visiting professor fellowship and H.H.G. is also grateful to Fudan University for a visiting professor fellowship.

[1] R. Aebersold, M. Mann, Nature 2003, 422, 198.

[2] S. Vorderwülbecke, S. Cleverley, S. R. Weinberger, A. Wiesner, Nat. Methods 2005, 2, 3 .

[3] H. Y. Bi, L. Qiao, J. M. Busnel, V. Devaud, B. H. Liu, H. H. Girault, Anal. Chem. 2009, 81, 1177.

[4] M. Karas, R. Kruger, Chem. Rev. 2003, 103, 427.

[5] R. Knochenmuss, Analyst 2006, 131, 966.

[6] R. Knochenmuss, R. Zenobi, Chem. Rev. 2003, 103, 441.

[7] D. Robert, Catal. Today 2007, 122, 20.

[8] D. S. Zhang, J. A. Downing, F. J. Knorr, J. L. McHale, J. Phys. Chem. B 2006, 110, 21890.

[9] K. Szacilowski, W. Macyk, A. Drzewiecka-Matuszek, M. Brindell, G. Stochel, Chem. Rev. 2005, 105, 2647.

[10] B. O'Regan, M. Gratzel, Nature 1991, 353, 737.

[11] M. Ni, M. K. H. Leung, D. Y. C. Leung, K. Sumathy, Renewable Sustainable Energy Rev. 2007, 11, 401.

[12] A. L. Linsebigler, G. Q. Lu, J. T. Yates, Chem. Rev. 1995, 95, 735.

[13] G. Palmisano, V. Augugliaro, M. Pagliaro, L. Palmisano, Chem. Commun. 2007, 3425.

[14] L. Qiao, C. Roussel, J. J. Wan, J. Kong, P. Y. Yang, H. H. Girault, B. H. Liu, Angew. Chem. 2008, 120, 2686; Angew. Chem. Int. Ed. 2008, 47, 2646.

[15] L. Qiao, H. Y. Bi, J. Busnel, B. H. Liu, H. H. Girault, Chem. Commun. 2008, 6357.

[16] I. A. Shkrob, M. C. Sauer, D. Gosztola, J. Phys. Chem. B 2004, 108, 12512.

[17] M. S. Thompson, W. D. Cui, J. P. Reilly, Angew. Chem. 2004, 116 , 4895; Angew. Chem. Int. Ed. 2004, 43, 4791.

[18] M. S. Thompson, W. Cui, J. P. Reilly, in ASMS, USA, 2004 
[19] F. Kjeldsen, O. A. Silivra, I. A. Ivonin, K. F. Haselmann, M. Gorshkov, R. A. Zubarev, Chem. Eur. J. 2005, 11, 1803.

[20] K. F. Haselmann, B. A. Budnik, F. Kjeldsen, M. L. Nielsen, J. V. Olsen, R. A. Zubarev, Eur. J. Mass Spectrom. 2002, 8, 117.

[21] B. A. Budnik, K. F. Haselmann, R. A. Zubarev, Chem. Phys. Lett. 2001, 342, 299.

[22] R. A. Zubarev, D. M. Horn, E. K. Fridriksson, N. L. Kelleher, N. A. Kruger, M. A. Lewis, B. K. Carpenter, F. W. McLafferty, Anal. Chem. 2000, 72, 563.

[23] L. M. Mikesh, B. Ueberheide, A. Chi, J. J. Coon, J. E. P. Syka, J. Shabanowitz, D. F. Hunt, Biochim. Biophys. Acta Proteins Proteomics 2006, 1764,1811

[24] M. Takayama, J. Am. Soc. Mass Spectrom. 2001, 12, 420.

[25] R. G. Cooks, J. Mass Spectrom. 1995, 30, 1215.

[26] K. Demeure, L. Quinton, V. Gabelica, E. De Pauw, Anal. Chem. 2007, 79, 8678

[27] J. Chen, D. F. Ollis, W. H. Rulkens, H. Bruning, Water Res. 1999, 33 , 1173
[28] M. Jonsson, D. D. M. Wayner, D. A. Armstrong, D. K. Yu, A. Rauk, J. Chem. Soc. Perkin Trans. 2 1998, 1967.

[29] M. L. Huang, A. Rauk, J. Phys. Org. Chem. 2004, 17, 777.

[30] E. J. Rudd, S. D. Ross, M. Finkelst, J. Org. Chem. 1972, 37, 1763.

[31] S. D. Ross, M. Finkelst, R. C. Petersen, J. Am. Chem. Soc. 1966, 88, 4657.

[32] S. D. Ross, M. Finkelstein, R. C. Petersen, J. Am. Chem. Soc. 1964, 86, 2745.

[33] S. D. Ross, M. Finkelst, R. C. Petersen, J. Org. Chem. 1966, 31, 128

[34] C. Galli, P. Gentili, O. Lanzalunga, Angew. Chem. 2008, 120, 4868; Angew. Chem. Int. Ed. 2008, 47, 4790.

[35] F. Recupero, C. Punta, Chem. Rev. 2007, 107, 3800.

[36] G. Rothenberger, J. Moser, M. Gratzel, N. Serpone, D. K. Sharma, J. Am. Chem. Soc. 1985, 107, 8054 .

[37] L. Qiao, C. Roussel, J. J. Wan, P. Y. Yang, H. H. Girault, B. H. Liu, J. Proteome Res. 2007, 6, 4763.

Received: October 28, 2008 Revised: February 17, 2009 Published online: June 2, 2009 